\title{
Electron Microscopy and Spectroscopy of Citrate Induced Calcium Oxalate Crystal Structure and Hydration State Changes, and Implications for Kidney Stones
}

\author{
David J. Banner ${ }^{1}$, Emre Firlar ${ }^{1,2}$, Jodi K. Finlay ${ }^{1}$, Reza Shahbazian-Yassar ${ }^{2}$, and Tolou Shokuhfar ${ }^{1}$. \\ 1. The University of Illinois at Chicago, Department of Bioengineering, Chicago, Illinois, USA \\ 2. The University of Illinois at Chicago, Department of Mechanical and Industrial Engineering, Chicago, \\ Illinois, USA
}

In the USA alone, 20 million people are currently have kidney stones (KS) or are predicted to develop $\mathrm{KS}$ during their lifetimes. The most common KS symptom is excruciating pain, but symptoms often include nausea and emesis, blood in urine, difficulty urinating, and frequent urination. KS may require removal of the kidney and are associated with Chronic Kidney Disease as well as Cardiovascular Diseases [1]. Approximately $80 \%$ of KS contain Calcium Oxalate (CaOx) as the major phase with $\mathrm{CaOx}$ monohydrate (COM) as the most prominent hydration state [2]. Therefore, medical KS research primarily focuses on $\mathrm{CaOx}$ biomineralization and potential $\mathrm{CaOx}$ treatments.

Citrate, a common KS treatment and dietary inhibitor, was previously thought to function by lowering the effective $\mathrm{CaOx}$ supersaturation within the urine. However, in vitro studies of both human and artificial urine have shown that addition and supersaturation of $\mathrm{CaOx}$ does not cause $\mathrm{CaOx}$ biomineralization [3]. This finding suggests a far more complex mechanism by which citrate and other $\mathrm{KS}$ treatments prevent formation of $\mathrm{CaOx} \mathrm{KS}$. Insight into the mechanism or mechanisms by which citrate inhibits $\mathrm{CaOx}$ biomineralization would allow optimization of medical treatment protocols and discernment of the desired properties of improved next-generation KS treatments.

$\mathrm{CaOx} \mathrm{KS}$ research has taken two broad approaches: 1) in vitro studies of $\mathrm{CaOx}$ crystallization within human or synthetic urine, and 2) organismal in vivo studies and clinical studies. These approaches are essential for determining the efficacy of a $\mathrm{CaOx} \mathrm{KS}$ treatment. However, these approaches do not provide fundamental data on the manner in which $\mathrm{CaOx}$ treatments affect the formation of $\mathrm{CaOx}$. This study implements nanoscale resolution electron microscopy techniques, including High Resolution Transmission Electron Microscopy (HR-TEM), Energy Dispersive X-Ray Spectroscopy (EDS) elemental analysis, Electron Energy Loss Spectroscopy (EELS) chemical analysis, and Select Area Electron Diffraction (SAED) analysis of crystal structures to provide a fundamental understanding of the formation of $\mathrm{CaOx}$ mineralization and the influence of citrate.

To determine the impact of citrate on $\mathrm{CaOx}$ solubility and crystal structure, control $\mathrm{CaOx}$ samples were synthesized by chemical reaction between $0.125 \mathrm{M}: 0.125 \mathrm{M}$ sodium oxalate $(\mathrm{NaOx})$ and calcium chloride $\left(\mathrm{CaCl}_{2}\right)$, while $\mathrm{CaOx}$ experimental samples were synthesized by chemical reaction between $0.125 \mathrm{M}: 0.125 \mathrm{M} \mathrm{NaOx}$ and $\mathrm{CaCl}_{2}$ in the presence of $1 \mathrm{M}$ citrate. This citrate concentration is substantially higher than medically achievable concentrations. In each experiment, six control $\mathrm{CaOx}$ samples and six citrate treated $\mathrm{CaOx}$ samples were synthesized. A control sample and an experimental sample were analyzed after the solutions settled for 30 minutes, and other samples were subsequently analyzed at 3,6,12, 24, and 72 hours. At each time point, the supernatant was removed by pipette without disturbing the sample pellet. The remaining sample was then centrifuged and any remaining supernatant removed by pipette. The sample was then rinsed with ultrapure water and dried by evaporation. Alterations in the final weight of $\mathrm{CaOx}$ as a result of the rinsing procedure are minimal due to the $0.67 \mathrm{mg} / \mathrm{L}$ solubility of $\mathrm{CaOx}$ in water, while the more soluble sodium chloride byproducts and 
any dissolved calcium or oxalate are removed.

Compared to control $\mathrm{CaOx}$ synthesized without citrate, citrate treated $\mathrm{CaOx}$ showed no significant change in the total weight synthesized. EDS analysis of the sample (Figure 1) showed that samples were not contaminated by sodium or chloride. However, SAED (not shown) and HR-TEM (Figure 2) showed alterations in the crystal structure from monoclinic $\mathrm{COM}$ in the untreated $\mathrm{CaOx}$ samples to tetragonal $\mathrm{CaOx}$ dihydrate (COD) in the citrate treated $\mathrm{CaOx}$ samples.

The preliminary results shown here suggest that citrate may act by influencing the crystallization of $\mathrm{CaOx}$ to a less mechanically stable structure. Furthermore, a previous study by Gan et al. showed that COM better adheres to cell walls as compared to COD [4]. This suggests that citrate may inhibit the formation of KS by altering the crystal structure and preventing cell adherence [5].

\section{References:}

[1] Rule, AD. et al. Journal of the American Society of Nephrology. 21 (2010), p. 1641.

[2] Omar, M. Monga, M.; Calle, JC. J. Nephrol. Ther. 5 (2015), p. 198.

[3] Kavanagh, JP. Jones, L. Rao, PN. Urological Research. 27 (1999), p. 231.

[4] Gan, Q. et al. Int. J. Nanomedicine 11 (2016), p. 2839.

[5] The authors acknowledge funding from the National Science Foundation CAREER Award, grant No. DMR 1564950. This work made use of instruments in the Electron Microscopy Service (Research Resources Center, UIC)



Figure 1. Energy Dispersive X-Ray Spectroscopy mapping of $\mathrm{CaOx}$ synthesized by reaction between sodium oxalate and calcium chloride. The sample was prepared and rinsed as described in the methods detailed in this paper. A) shows the distribution of oxygen, while B) shows the calcium distribution with the sample shown in C). C), shows a High Angle Angular Dark Field Scanning Transmission Electron Microscopy (STEM) image of the sample. D), shows the total elemental content of the selected area. Notably, possible sodium and chloride are absent. Other elements, including aluminum, chromium, and copper are byproducts of STEM and were not introduced to the solution during the sample synthesis process. Imaged with the JEOL ARM-220CF at the University of Illinois Research Resources Center.

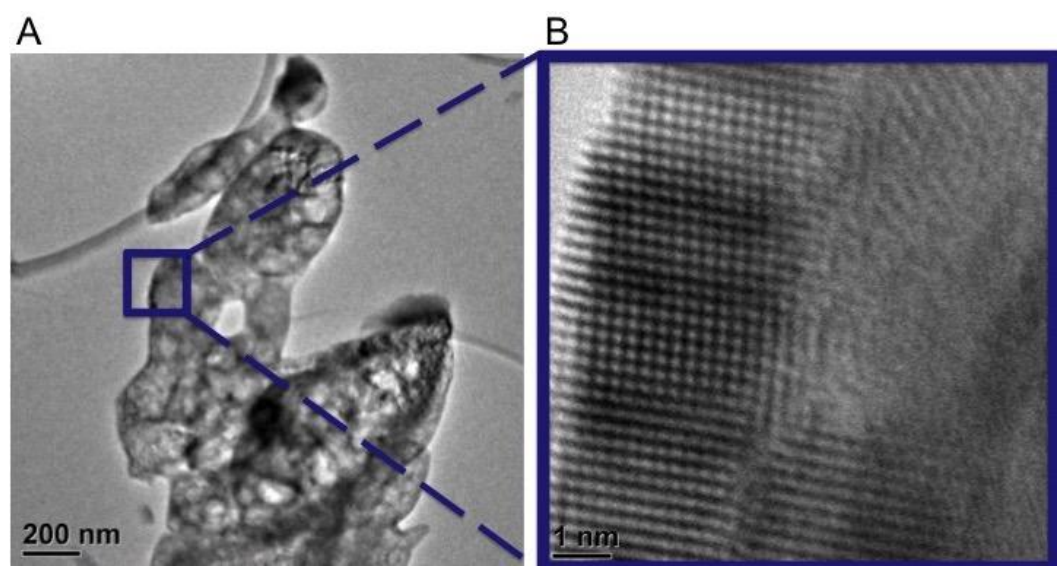

Figure 2. Transmission Electron Microscopy (TEM) of the $\mathrm{CaOx}$ synthesized by reaction between sodium oxalate and calcium chloride. The sample was prepared and rinsed as described in the methods detailed in this paper. A), shows TEM imaging of the sample. B), shows High Resolution TEM of the sample. In the left portion of B), individual atoms are visible. The right portion of the image is out of atomic resolution focus due to the thickness of the sample and the difference in height. By measuring the contrast across the visible atoms, the lattice spacing can be determined. It measures $2.7 \AA$, confirming the Select Area Electron Diffraction (not shown). Imaged with the JEOL 3010 TEM at the University of Illinois Research Resources Center. 